\title{
A SEARCH FOR COMPANIONS TO BROWN DWARFS IN THE TAURUS AND CHAMAELEON STAR-FORMING REGIONS*
}

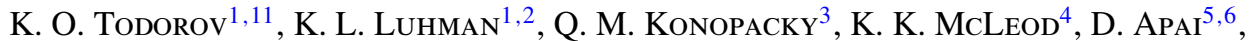 \\ A. M. Ghez ${ }^{7,8}$, I. PascuCCI ${ }^{5,6}$, AND M. Robberto ${ }^{9,10}$ \\ ${ }^{1}$ Department of Astronomy and Astrophysics, The Pennsylvania State University, University Park, PA 16802, USA \\ ${ }^{2}$ Center for Exoplanets and Habitable Worlds, The Pennsylvania State University, University Park, PA 16802, USA \\ ${ }^{3}$ Lawrence Livermore National Laboratory, 7000 East Avenue, Livermore, CA 94550, USA \\ ${ }^{4}$ Whitin Observatory, Wellesley College, Wellesley, MA 02481, USA \\ ${ }^{5}$ Department of Astronomy, University of Arizona, 933 N. Cherry Avenue, Tucson, AZ 85721, USA \\ ${ }^{6}$ Lunar and Planetary Laboratory, 1629 E. University Blvd., Tucson, AZ 85721, USA \\ ${ }^{7}$ Division of Astronomy and Astrophysics, University of California, Los Angeles, CA 90095, USA \\ ${ }^{8}$ Institute of Geophysics and Planetary Physics, University of California, Los Angeles, CA 90095, USA \\ ${ }^{9}$ Space Telescope Science Institute, 3700 San Martin Drive, Baltimore, MD 21218, USA \\ ${ }^{10}$ Center for Astrophysical Sciences, Johns Hopkins University, 3400 North Charles Street, Baltimore, MD 21218, USA \\ Received 2013 December 7; accepted 2014 April 1; published 2014 May 20
}

\begin{abstract}
We have used WFPC2 on board the Hubble Space Telescope to obtain images of 47 members of the Taurus and Chamaeleon I star-forming regions that have spectral types of M6-L0 $\left(M \sim 0.01-0.1 M_{\odot}\right)$. An additional late-type member of Taurus, FU Tau (M7.25+M9.25), was also observed with adaptive optics at Keck Observatory. In these images, we have identified promising candidate companions to 2MASS J04414489+2301513 $(\rho=0$ '! 105/15 AU), 2MASS J04221332+1934392 ( $\left.\rho=00^{\prime} 05 / 7 \mathrm{AU}\right)$, and ISO $217\left(\rho=00^{\prime} 03 / 5 \mathrm{AU}\right)$. We reported the first candidate in a previous study, showing that it has a similar proper motion as the primary in images from WFPC 2 and Gemini adaptive optics. We have collected an additional epoch of data with Gemini that further supports that result. By combining our survey with previous high-resolution imaging in Taurus, Chamaeleon I, and Upper Sco ( $\tau \sim 10 \mathrm{Myr})$, we measure binary fractions of $14 / 93=0.15_{-0.03}^{+0.05}$ for M4-M6 $\left(M \sim 0.1-0.3 M_{\odot}\right)$ and 4/108 $=0.04_{-0.01}^{+0.03}$ for $>$ M6 $\left(M \lesssim 0.1 M_{\odot}\right)$ at separations of $>10 \mathrm{AU}$. Given the youth and low density of these regions, the lower binary fraction at later types is probably primordial rather than due to dynamical interactions among association members. The widest low-mass binaries ( $>100 \mathrm{AU}$ ) also appear to be more common in Taurus and Chamaeleon I than in the field, which suggests that the widest low-mass binaries are disrupted by dynamical interactions at $>10 \mathrm{Myr}$, or that field brown dwarfs have been born predominantly in denser clusters where wide systems are disrupted or inhibited from forming.
\end{abstract}

Key words: binaries: visual - brown dwarfs - stars: formation - stars: low-mass - stars: pre-main sequence

Online-only material: machine-readable table

\section{INTRODUCTION}

As with stars at higher masses, the binary properties of lowmass stars and brown dwarfs may provide insight into their formation and dynamical evolution (Duchêne \& Kraus 2013). Multiplicity at low masses has been characterized primarily through high-resolution imaging in the solar neighborhood (Koerner et al. 1999; Martín et al. 1999; Reid et al. 2001; Bouy et al. 2003; Burgasser et al. 2003; Close et al. 2003) and nearby young clusters and associations (Neuhäuser et al. 2002; Martín et al. 2003; Luhman et al. 2005b; Kraus et al. 2005, 2006; Konopacky et al. 2007; Biller et al. 2011; Kraus \& Hillenbrand 2012). These surveys have found that the binary fractions and the separations of binaries decrease and the mass ratios increase from stars to brown dwarfs (Burgasser et al. 2007; Kraus \& Hillenbrand 2012). Although most binary brown dwarfs have small separations $(a<20 \mathrm{AU})$, a few wide systems have

\footnotetext{
* Based on observations performed with the NASA/ESA Hubble Space Telescope, Gemini Observatory, and the W. M. Keck Observatory. The Hubble observations are associated with proposal IDs 11203, 11204, and 11983 and were obtained at the Space Telescope Science Institute, which is operated by the Association of Universities for Research in Astronomy, Inc., under NASA contract NAS 5-26555.

${ }^{11}$ Current address: Institute for Astronomy, ETH Zurich, Wolfgang-PauliStrasse 27, CH-8093 Zurich, Switzerland; todorovk@ phys.ethz.ch.
}

been uncovered (Luhman 2004a; Chauvin et al. 2004). The low binding energies of these wide binaries would tend to suggest that dynamical interactions did not play a role in their formation (Reipurth \& Clarke 2001), although a few ejected brown dwarfs may be captured into wide systems in denser clusters (Bate \& Bonnell 2005).

The dependence of the binary properties of low-mass stars and brown dwarfs on age and star-forming environment is not well-constrained by existing data. As a result, it is unclear whether wide low-mass binaries are frequently disrupted by interactions with stars, either in their natal clusters or in the Galactic field, and how the initial conditions of star formation influence binarity at low masses. The Taurus and Chamaeleon I star-forming regions are promising sites for providing some of the data needed to investigate these issues. They are among the nearest star-forming regions (140 and $165 \mathrm{pc}$ ), young enough that dynamical interactions are minimized (1 and 2-3 Myr), most of their members have relatively low extinction $\left(A_{V} \lesssim 4\right)$, and they have been searched thoroughly for substellar members (Kenyon et al. 2008; Luhman 2008). These two regions also offer the opportunity for characterizing the multiplicity produced by low-density star-forming conditions, which can be compared to measurements in richer and denser clusters at the same age as well as older populations in open clusters and the solar neighborhood. 
High-resolution imaging has previously been applied to low-mass members of Taurus and Chamaeleon I (Neuhäuser et al. 2002; Kraus et al. 2006; Ahmic et al. 2007; Konopacky et al. 2007; Luhman 2007; Lafrenière et al. 2008; Kraus \& Hillenbrand 2012), which has included 45 primaries with spectral types later than M6 $\left(M \lesssim 0.1 M_{\odot}\right)$. To improve upon the statistical accuracy of those multiplicity measurements, we have performed an imaging survey that includes most of the remaining known late-type members of Taurus and Chamaeleon I using the Hubble Space Telescope (HST) and Keck Observatory, resulting in a sample of 73 primaries in these regions later than M6 for which high-resolution data are now available. In this paper, we describe the sample selection and observing strategy in our survey (Section 2) and our analysis of the resulting images (Section 3). We then identify the most promising candidate companions in our data and combine our sample with those of previous surveys to measure the binary fraction as a function of spectral type (Section 4). We conclude by discussing the implications of our survey for measurements of multiplicity at low masses and for the formation of brown dwarfs (Section 5).

\section{OBSERVATIONS}

\subsection{Hubble Images}

\subsubsection{Sample Selection}

We have obtained most of the data in our survey with the Wide Field Planetary Camera 2 (WFPC2) on board HST. In our proposal for WFPC2 imaging in Taurus, we selected all members of this region that had spectral types later than M6, which had not been previously imaged with HST (Kraus et al. 2006), and that were known as of early 2007, which corresponded to 32 objects. However, observations could not be scheduled for three of the targets, 2MASS J04325026+2422115, 2MASS $\mathrm{J} 04335245+2612548,{ }^{12}$ and 2MASS J04380083+2558572. ${ }^{13}$ We replaced these sources with two Taurus members that have spectral types of M6 (2MASS J04350850+2311398, 2MASS J04400067+2358211) and a new member later than M6 that we uncovered after the submission of the original proposal (2MASS J04373705+2331080; Luhman et al. 2009a). Our WFPC2 images of these 32 targets encompassed 4 additional members of Taurus, FM Tau, V773 Tau, CW Tau, and 2MASS J04414565+2301580, all of which were saturated. One of our targets has previously been observed with speckle imaging (2MAS J04442713+2512164; Konopacky et al. 2007). That object and 18 other targets have been observed with AO imaging (Kraus \& Hillenbrand 2012).

For the WFPC2 sample in Chamaeleon I, we selected all known members that are later than M6 and that have not been previously observed with HST (Neuhäuser et al. 2002; Luhman 2007) with the exception of 2MASS J11195652-7504529 and 2MASS J11070369-7724307, which were omitted because the former is in the outskirts of the cluster and the latter is highly reddened. The resulting sample contained 19 objects. The observations of one target failed (ESO H $\alpha$ 554) and three targets were not observed before the decommissioning of WFPC2 (2MASS J11085176-7632502, 2MASS J11104006-7630547, ISO $138^{14}$ ). Thus, we were able to obtain WFPC2 images of 15

\footnotetext{
12 This object has been observed with adaptive optics (AO) imaging (Kraus \& Hillenbrand 2012).

13 This object has been observed with speckle imaging (Konopacky et al. 2007).

14 This object has been observed with AO imaging (Lafrenière et al. 2008).
}

late-type primaries in Chamaeleon I. OTS 32, C1-2, 2MASS J11011926-7732383 B, CHXR 84, and Hn11 also fell within the field of view of these data. The latter two stars were saturated. One of our 15 targets, CHSM 17173, has been observed with AO imaging (Lafrenière et al. 2008).

We present in Table 1 the sample of 47 late-type primaries in Taurus and Chamaeleon I that we have observed with WFPC2. We also include the extra members that appeared within the images and that were not saturated (OTS 32, C1-2, 2MASS $\mathrm{J} 11011926-7732383 \mathrm{~B})$ as well as the new companion to 2MASS J04414489+2301513 (hereafter 2M J044144) that we found in this survey and reported in an earlier study (Todorov et al. 2010).

To obtain high-resolution images for two late-type members of Chamaeleon I that have high extinctions and thus were inaccessible with WFPC2, we used the Near-Infrared Camera and Multi-Object Spectrometer (NICMOS) on board HST. These objects consisted of 2MASS J11070369-7724307 (M7.5) and 2MASS J11062942-7724586 (M6).

\subsubsection{Observing Strategy}

We obtained the WFPC2 images through the F791W and F850LP filters, which are similar to the Cousins $I$ and SDSS $z^{\prime}$ filters, respectively. We selected these filters because they provide the optimum combination of sensitivity to cool companions and spatial resolution and they produce a color-magnitude diagram that is effective in distinguishing young low-mass objects from field stars. WFPC2 contained four $800 \times 800$ CCDs. The plate scales of the PC and the three WFC arrays were 0. '046 pixel $^{-1}$ and 0 '. 1 pixel $^{-1}$, respectively. To mitigate the effects of degraded charge-transfer efficiency (CTE), we placed each target near the readout amplifier in the PC array. Each target was observed with a two-point dither pattern. At a given dither position, we obtained two images in each of the two filters. We selected three combinations of exposure times and gains for three ranges of optical magnitudes in order to avoid saturation of the targets. From the faintest to brightest targets, we used (1) $\tau_{791}=260 \mathrm{~s}, \tau_{850}=160 \mathrm{~s}$, gain $=7$, (2) $\tau_{791}=260 \mathrm{~s}$, $\tau_{850}=160 \mathrm{~s}$, gain $=15$, and (3) $\tau_{791}=200 \mathrm{~s}, \tau_{850}=160 \mathrm{~s}$, gain $=15$. These exposure times apply to each of the four individual images for a given filter. For the third set of exposure times, we also included a pair of dithered images with exposure times of $40 \mathrm{~s}$ in F791W to provide unsaturated data for the center of the point-spread function (PSF). We did not specify the position angle of the camera on the sky for these observations. Each object was observed during one orbit, corresponding to a total of 47 orbits.

The NICMOS observations were performed with the NIC2 camera and the F110W, F160W, and F205W filters. The camera contained a $256 \times 256$ array that had a plate scale of 0 .'075 pixel $^{-1}$. We obtained one image in each filter at each position in a six-point dither pattern that was centered in the NIC2 array. The exposure times of the individual images were 128, 128, and $96 \mathrm{~s}$ in F110W, F160W, and F205W, respectively. Immediately before and after the dither sequence on each target, we collected one image in F205W at a position 30" from the target to measure the background. Each of the two NICMOS targets was observed during one orbit.

\subsection{Keck Images}

Additional late-type members of Taurus have been uncovered since the planning of our HST observations. We obtained highresolution images of two of these objects, FU Tau A and B 
Table 1

Members of Taurus and Chamaeleon I in the WFPC2 Images

\begin{tabular}{|c|c|c|c|c|c|}
\hline $2 \mathrm{MASS}^{\mathrm{a}}$ & Other Name & Spectral Type & $m_{791} \mathrm{~b}$ & $m_{850}{ }^{b}$ & Date \\
\hline J04141188+2811535 & $\cdots$ & M6.25 & 17.35 & 16.45 & 2008 Nov 8 \\
\hline J04152409+2910434 & $\cdots$ & M7 & 18.22 & 17.12 & 2008 Aug 1 \\
\hline J04161885+2752155 & $\ldots$ & M6.25 & 16.72 & 15.76 & 2007 Sep 15 \\
\hline J04201611+2821325 & $\ldots$ & M6.5 & 17.64 & 16.72 & 2007 Oct 24 \\
\hline $\mathrm{J} 04215450+2652315$ & $\cdots$ & M8.5 & 20.85 & 19.40 & 2008 Jul 31 \\
\hline $\mathrm{J} 04221332+1934392 \mathrm{~A}+\mathrm{B}$ & $\cdots$ & M8 & $17.33^{\mathrm{c}}$ & $16.25^{\mathrm{c}}$ & 2007 Aug 6 \\
\hline J04221644+2549118 & $\ldots$ & M7.75 & 17.39 & 16.34 & 2008 Aug 1 \\
\hline $\mathrm{J} 04242090+2630511$ & $\cdots$ & M6.5 & 17.35 & 16.47 & 2008 Aug 1 \\
\hline $\mathrm{J} 04263055+2443558$ & $\cdots$ & M8.75 & 19.54 & 18.26 & 2008 Aug 9 \\
\hline J04270739+2215037 & $\ldots$ & M6.75 & 16.02 & 15.23 & 2008 Aug 2 \\
\hline $\mathrm{J} 04274538+2357243$ & $\cdots$ & M8.25 & 19.59 & 18.39 & 2008 Aug 1 \\
\hline $\mathrm{J} 04290068+2755033$ & $\cdots$ & M8.25 & 18.45 & 17.28 & 2008 Aug 15 \\
\hline J04302365+2359129 & $\ldots$ & M8.25 & 19.57 & 18.39 & 2008 Aug 6 \\
\hline $\mathrm{J} 04311907+2335047$ & $\cdots$ & M7.75 & 18.19 & 17.02 & 2008 Aug 6 \\
\hline $\mathrm{J} 04312669+2703188$ & $\cdots$ & M7.5 & 19.55 & 18.34 & 2008 Aug 10 \\
\hline $\mathrm{J} 04320329+2528078$ & $\ldots$ & M6.25 & 15.51 & sat & 2008 Aug 15 \\
\hline $\mathrm{J} 04322329+2403013$ & $\cdots$ & M7.75 & 16.48 & 15.49 & 2008 Sep 7 \\
\hline $\mathrm{J} 04334291+2526470$ & $\cdots$ & M8.75 & 19.55 & 18.24 & 2008 Aug 12 \\
\hline $\mathrm{J} 04350850+2311398$ & $\ldots$ & M6 & 16.27 & 15.46 & 2007 Oct 6 \\
\hline J04354526+2737130 & $\cdots$ & M9.25 & 19.84 & 18.54 & 2008 Aug 4 \\
\hline $\mathrm{J} 04361030+2159364$ & $\cdots$ & M8.5 & 19.43 & 18.28 & 2008 Aug 15 \\
\hline $\mathrm{J} 04373705+2331080$ & $\ldots$ & L0 & 23.01 & 21.62 & 2007 Aug 25 \\
\hline J04385871+2323595 & $\cdots$ & M6.5 & 16.07 & sat & 2008 Aug 4 \\
\hline J04390396+2544264 & $\cdots$ & M7.25 & 16.95 & 15.93 & 2008 Aug 6 \\
\hline $\mathrm{J} 04390637+2334179$ & $\cdots$ & M7.5 & 15.71 & sat & 2008 Aug 18 \\
\hline J04400067+2358211 & $\cdots$ & M6 & 16.17 & 15.42 & 2007 Aug 24 \\
\hline J04414489+2301513A & $\cdots$ & M8.5 & 18.93 & 17.85 & 2008 Aug 20 \\
\hline J04414489+2301513B & $\cdots$ & $\ldots$ & 21.16 & 19.91 & 2008 Aug 20 \\
\hline J04414825+2534304 & $\ldots$ & M7.75 & 18.52 & 17.44 & 2007 Oct 27 \\
\hline J04442713+2512164 & IRAS 04414+2506 & M7.25 & 16.45 & 15.44 & 2007 Aug 19 \\
\hline J04484189+1703374 & $\ldots$ & M7 & sat & 16.59 & 2007 Aug 8 \\
\hline $\mathrm{J} 04552333+3027366$ & $\cdots$ & M6.25 & 16.99 & 16.16 & 2007 Aug 26 \\
\hline J04574903+3015195 & $\ldots$ & M9.25 & 20.73 & 19.43 & 2007 Aug 26 \\
\hline J11011926-7732383A & $\cdots$ & M7.25 & 18.16 & 17.02 & 2009 Apr 30 \\
\hline J11011926-7732383B & $\cdots$ & M8.25 & 19.30 & 18.00 & 2009 Apr 30 \\
\hline J11020610-7718079 & $\cdots$ & M8 & 20.65 & 19.35 & 2009 Mar 16 \\
\hline J11025374-7722561 & $\ldots$ & M8.5 & 20.96 & 19.64 & 2009 Mar 4 \\
\hline$\cdots$ & Cha J11062854-7618039 & M9 & 21.98 & 20.72 & 2009 Apr 24 \\
\hline$\ldots$ & Cha J11070768-7626326 & L0 & 22.75 & 21.46 & 2009 Apr 24 \\
\hline J11082570-7716396 & $\cdots$ & M8 & 21.69 & 20.38 & 2009 Apr 23 \\
\hline J11084952-7638443 & $\ldots$ & M8.75 & 20.95 & 19.64 & 2009 Apr 21 \\
\hline $\mathrm{J} 11095215-7639128 \mathrm{~A}+\mathrm{B}$ & ISO $217 \mathrm{~A}+\mathrm{B}$ & M6.25 & $19.18^{\mathrm{c}}$ & $18.12^{\mathrm{c}}$ & 2009 Apr 26 \\
\hline J11095505-7632409 & C1-2 & $\ldots$ & 20.33 & 19.23 & 2009 Apr 23 \\
\hline J11100336-7633111 & OTS 32 & M4 & 22.44 & 21.45 & 2009 Apr 23 \\
\hline J11100658-7642486 & $\ldots$ & M9.25 & 21.67 & 20.31 & 2009 Apr 24 \\
\hline $\mathrm{J} 11100934-7632178$ & OTS 44 & M9.5 & 22.11 & 20.64 & 2009 Apr 23 \\
\hline J11102226-7625138 & CHSM 17173 & M8 & 17.95 & 16.88 & 2009 Apr 27 \\
\hline J11112249-7745427 & $\ldots$ & M8.25 & 20.05 & 18.86 & 2009 Apr 24 \\
\hline $\mathrm{J} 11114533-7636505$ & $\cdots$ & M8 & 19.89 & 18.75 & 2009 Feb 27 \\
\hline $\mathrm{J} 11122250-7714512$ & $\cdots$ & M9.25 & 20.74 & 19.40 & 2009 Apr 24 \\
\hline J11123099-7653342 & $\cdots$ & M7 & 18.14 & 17.21 & 2009 Mar 3 \\
\hline
\end{tabular}

Notes.

a 2MASS Point Source Catalog (Skrutskie et al. 2006).

b An entry of "sat" indicates that the object was saturated in this band.

c This photometry applies to the combined flux from this partially resolved binary (Figure 1). The flux ratio for the components of the binary is in Table 2 .

(Luhman et al. 2009b), with the near-infrared camera NIRC2 (PI: K. Matthews) in conjunction with the laser guide star AO system at the Keck II $10 \mathrm{~m}$ telescope (Wizinowich et al. 2006; van Dam et al. 2006). These observations were performed on 2008 December 18 and 2010 December 9. The target was bright enough to provide the tip/tilt correction. The plate scale of NIRC2 is $9.952 \pm 0.002$ mas pixel ${ }^{-1}$ and its columns are rotated by $0.252 \pm 0.009$ relative to the nominal position angle computed from the image headers (Yelda et al. 2010). We collected three $H$-band images at each position in a three-point 
dither pattern. The individual images consisted of 10 and 50 coadditions of $0.5 \mathrm{~s}$ exposures during the observations in 2008 and 2010 , respectively.

\subsection{Gemini Images}

Our analysis of the WFPC2 data in Section 3.2 reveals a candidate companion to 2M J044144. In Todorov et al. (2010), we obtained AO images of this pair to better constrain the nature of the candidate. The images were taken in $H$ and $K^{\prime}$ filters with the Gemini Near-Infrared Imager (NIRI; Hodapp et al. 2003) and the ALTAIR AO system at the Gemini North telescope. The plate scale was $0{ }^{\prime} .0214 \mathrm{pixel}^{-1}$ and the field of view was $22^{\prime \prime} \times 22^{\prime \prime}$. The relative positions of $2 \mathrm{M} \mathrm{J044144}$ and its candidate companion remained unchanged between the WFPC2 and AO observations, indicating that the candidate shares a similar proper motion with the primary and thus is a Taurus member rather than a field star. The tip-tilt star for the AO observations was 2MASS J04414565+2301580 (hereafter

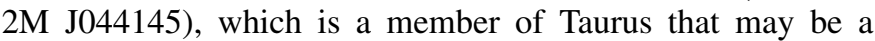
wide companion to $2 \mathrm{M} \mathrm{J044144} \mathrm{(12".3).} \mathrm{The} \mathrm{AO} \mathrm{images} \mathrm{from}$ Todorov et al. (2010) resolved a faint candidate companion at $0^{\prime \prime} .23$ from that star, which could make 2M J044144 and $2 \mathrm{M} \mathrm{J044145}$ a quadruple system. To assess this possibility, we sought constraints on the relative proper motions of the four objects by observing them again with NIRI+ALTAIR. The data were collected on the night of 2011 February 13. The observing strategy was the same as for the first epoch from Todorov et al. (2010) except that one dither sequence was performed in each filter instead of two.

\section{IMAGE ANALYSIS}

\subsection{Reduction of Hubble Images}

We employed the MultiDrizzle software package (Koekemoer et al. 2002) for performing cosmic ray rejection on the WFPC2 images and for combining the dithered frames for a given filter and exposure time. We adopted a drop size of 0.85 native pixels and resampled plate scales of $0{ }^{\prime} .01$ pixel $^{-1}$ and 0.05 pixel $^{-1}$ for the PC and WFC images, respectively. Using the IRAF routine starfind, we identified all point sources in each of the reduced PC and WFC images. Spurious detections were manually removed through visual inspection of the images. To search for sources that are blended with the primaries targeted by our survey, we applied PSF subtraction, as described in the next section.

We measured aperture photometry for all unsaturated point sources using the IRAF task phot with an aperture radius of 2 pixels on the native scale, corresponding to $0^{\prime \prime} .091$ and 0.2 for the PC and WFC images, respectively. We estimated aperture corrections between these radii and an aperture of 0.5 for each array and filter using stars that were isolated, bright, and unsaturated. The average values were 0.22 (F791W/WFC), 0.24 (F850LP/WFC), 0.54 (F791W/PC), and $0.61 \mathrm{mag}$ (F850LP/ $\mathrm{PC})$. The aperture correction from 0.5 to an infinite aperture is $0.1 \mathrm{mag}$. We arrived at the final photometric magnitudes by combining the measurements from phot with the aperture corrections, CTE corrections, ${ }^{15}$ and the zero point fluxes from the image headers. The CTE corrections were larger for fainter objects. Since all of the primaries were well-detected, their CTE corrections were fairly small ( $\lesssim 0.1 \mathrm{mag})$. However, because of the advanced age of WFPC 2 at the time of our observations, the CTE corrections were quite large for the faintest objects

\footnotetext{
15 http://purcell.as.arizona.edu/wfpc2_calib
}

(0.5-1 mag). The magnitude at which saturation occurs is brighter in Taurus than in Chamaeleon I because short exposures were included for some of the former targets. The photometry for the known members of Taurus and Chamaeleon I within the WFPC2 images is provided in Table 1. The uncertainties in these measurements are $\sim 0.05 \mathrm{mag}$, which are dominated by the errors in the aperture corrections.

As with the WFPC2 data, we reduced the NICMOS images with MultiDrizzle. The resampled plate scale was $00^{\prime \prime} 025$ pixel $^{-1}$. The field of view of each reduced image was small enough $\left(22^{\prime \prime} \times 22^{\prime \prime}\right)$ that visual inspection was adequate for identifying sources in these images. In the F110W images, the only objects detected consisted of the two members of Chamaeleon I that were targeted. For both F160W and F205W, two and four additional sources were detected in the images of $2 \mathrm{MASS}$ J11070369-7724307 and 2MASS J11062942-7724586, respectively. One of these objects is Cha J11062788-7724543, which is 7" from 2MASS J11062942-7724586 and has been identified as a candidate low-mass protostar based on its red mid-infrared (IR) colors (Luhman et al. 2008). The other sources have separations of $>3^{\prime \prime}$ from the primaries. They are unlikely to be cluster members based on photometry from other telescopes (Luhman 2007; Luhman et al. 2008). Our analysis to check for marginally resolved companions is described in the next section.

\subsection{PSF Analysis of Hubble Images}

To detect sources at small angular separations from the survey primaries in the WPFC2 and NICMOS images, we performed PSF subtractions using the IMFITFITS software, written by Brian McLeod and described in Lehár et al. (2000). For each primary, we fit its PSF with the PSF of every other primary that was observed in that star-forming region. We then visually inspected each of the subtracted images and identified the ones with the smallest residuals. The PSFs of other primaries were better choices for PSF subtraction than other stars in the image of a given primary because all of the primaries have similar colors and were observed at the same location on the PC array. Synthetic PSF fitting (e.g., TinyTim) was not attempted since it provides poorer fits than observed stellar PSFs (Luhman et al. 2005b).

We found significant residuals after subtraction of a single PSF to 2M J044144, 2MASS J04221332+1934392, and ISO 217. PSF subtraction for 2M J044144 revealed a faint companion at a separation of 0 '. 105 , as described by Todorov et al. (2010). The other two objects appear to be marginally resolved binaries. To measure the relative positions and fluxes of the components of each system, we created a grid of subtracted images that used pairs of PSFs with a range of separations, position angles, and flux ratios. Through visual inspection of the residuals in this grid of subtracted images, we estimated the binary parameters and their uncertainties. These measurements are presented in Table 2. Figure 1 shows the images of 2MASS J04221332+1934392 and ISO 217 produced by subtraction of the best-fit single and double PSFs.

\subsection{Reduction and PSF Analysis of Keck Images}

The Keck NIRC2 data for FU Tau A and B were processed using standard reduction techniques for near-IR images. For each image, we subtracted a frame from another dither position to remove the sky background, applied a mask for bad pixels, divided by a flat field image, and corrected for optical distortion 


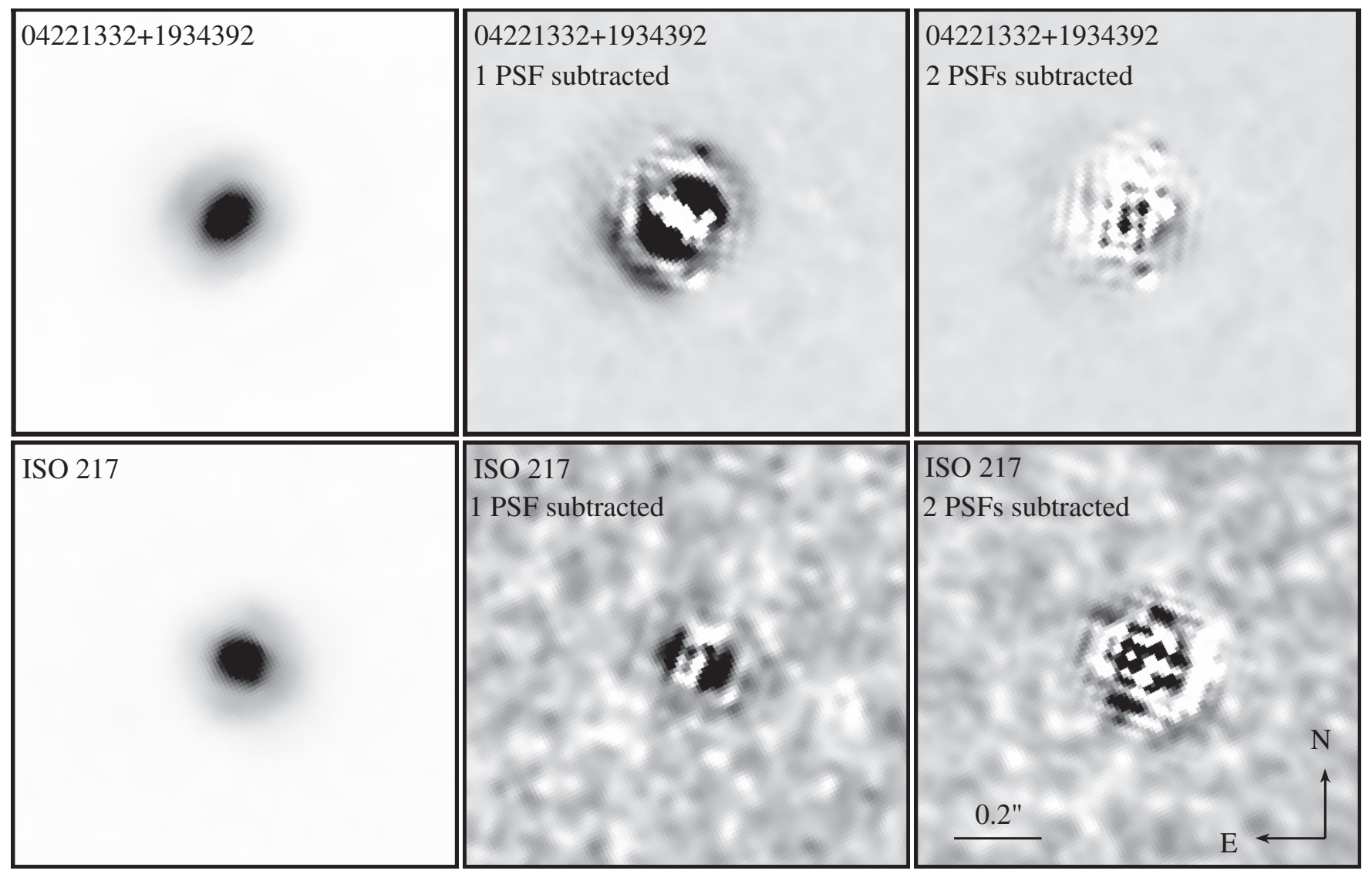

Figure 1. WFPC2 F791W images of the young late-type objects 2MASS J04221332+1934392 and ISO 217 before and after PSF subtraction. Large symmetric residuals remain after subtraction of single PSFs for both objects, indicating that they are marginally resolved binaries. The residuals are much smaller when each image is fit with a pair of PSFs. For each image prior to PSF subtraction, the maximum of the intensity scale is 50\% of the peak of the PSF. The upper limits for the scales in the PSF-subtracted images are 2\% and 1\% of the PSF peaks for 2MASS J04221332+1934392 and ISO 217, respectively.

Table 2

Astrometry and Photometry for Partially Resolved Binaries from WFPC2

\begin{tabular}{lcccc}
\hline \hline Name & $\rho$ & P.A. $^{\text {a }}$ & \multicolumn{2}{c}{ Flux Ratio $^{\text {b }}$} \\
\cline { 3 - 5 } & $(\operatorname{arcsec})$ & F791W & F850LP \\
\hline 2MASS J04221332+1934392 & $0.051 \pm 0.003$ & $316 \pm 4$ & $0.69 \pm 0.03$ & $0.89 \pm 0.03$ \\
ISO 217 & $0.031 \pm 0.004$ & $238 \pm 8$ & $0.64 \pm 0.05$ & $0.47 \pm 0.08$
\end{tabular}

Notes.

${ }^{\text {a }}$ Position angle of the secondary relative to the primary.

b The combined photometry of each binary is in Table 1.

with a model provided in the pre-ship review document ${ }^{16}$ using IRAF and IDL routines. The dithered frames were then registered and combined.

Given their separation of 5".7, FU Tau A and B were wellresolved from each other in the NIRC2 images. Each component appears unresolved in these data without an obvious additional companion. To check for marginally resolved companions, we applied the IDL package StarFinder to FU Tau A and B (Diolaiti et al. 2000), which has a deblend function designed to detect close pairs given a good empirical PSF. We used each component as the PSF for the other object. In addition, separate single sources were observed close enough in time to FU Tau A that we could use them as secondary checks of the results of StarFinder. Our StarFinder analysis of the 2008 data showed a secondary source at a separation of $\sim 2.5$ pixels (or $\sim 0^{\prime \prime} 025$ ) and a position angle of $\sim 9^{\circ}$ from FU Tau A with a flux ratio of $\sim 2$. In the

\footnotetext{
16 http://www2.keck.hawaii.edu/inst/nirc2/preship_testing.pdf
}

images from 2010, no source was detected at a comparable separation, which could be due to the difference in image quality on the two nights. The observing conditions during the night in 2008 were very good, with a Strehl ratio estimated from the PSF of $\sim 20 \%$ in the $H$ band. The conditions in 2010 were worse, yielding a Strehl ratio of only 5\%. Additional observations are necessary to determine definitively whether FU Tau A is a binary as implied by the data from 2008 . We treat it as unresolved for the purposes of this study.

Our NIRC2 data have provided relative positions for the components of FU Tau that are more accurate than previous measurements from seeing-limited ground-based images. We measured a separation of $5^{\prime \prime} \cdot 69 \pm 00^{\prime} 02$ and 5".69 \pm 0 '.01, a position angle of $122.75 \pm 0.24$ and $122.77 \pm 0.02$, and an $H$-band flux ratio of $21 \pm 16$ and $35 \pm 3$ in the 2008 and 2010 images, respectively. These astrometric measurements are consistent with the previous data from Luhman et al. (2009b). A comparison of the astrometry between 2008 and 2010 indicates 
Table 3

Astrometry for Components of 2MASS J04414489+2301513 and 2MASS J04414565+2301580

\begin{tabular}{lcc}
\hline \hline $\begin{array}{l}\rho \\
(\operatorname{arcsec})\end{array}$ & $\begin{array}{l}\text { P.A. }^{\mathrm{a}} \\
(\mathrm{deg})\end{array}$ & Date \\
\hline \multicolumn{3}{c}{ 2MASS J04414489+2301513 A and B } \\
\hline $0.105 \pm 0.004$ & $120.4 \pm 2.2$ & 2008 Aug 20 \\
$0.105 \pm 0.005$ & $120.7 \pm 2.6$ & 2009 Oct 13 \\
$0.104 \pm 0.006$ & $122.8 \pm 3.0$ & 2011 Feb 13 \\
\hline \multicolumn{3}{c}{ 2MASS J04414565+2301580 A and B } \\
\hline $0.226 \pm 0.004$ & $84.8 \pm 1.0$ & 2009 Oct 13 \\
$0.230 \pm 0.006$ & $84.6 \pm 1.4$ & 2011 Feb 13 \\
\hline 2 MASS J04414565+2301580 A and 2MASS J04414489+2301513 A \\
\hline $12.325 \pm 0.004$ & $238.0 \pm 0.1$ & 2009 Oct 13 \\
$12.325 \pm 0.006$ & $237.9 \pm 0.1$ & 2011 Feb 13 \\
\hline
\end{tabular}

Note. ${ }^{\text {a }}$ Position angle of the secondary relative to the primary.

that FU Tau A and B share the same proper motion at a level of $\sim 5$ mas $\mathrm{yr}^{-1}$, which further supports the membership of these objects in the same star-forming population, either as components of a binary system or as unrelated Taurus members that are seen in projection near each other (Luhman et al. 2009b).

\subsection{Reduction and PSF Analysis of Gemini Images}

Our new Gemini AO images of 2M J044144 and 2M J044145 were reduced and analyzed in the same manner as the first epoch from Todorov et al. (2010) except that the data were corrected for distortion with an IDL script provided by Chad Trujillo. The first epoch data were reprocessed with this distortion correction as well. The resulting measurements of separations and position angles between the components of 2M J044144 A/B and 2M $\mathrm{J} 044145 \mathrm{~A} / \mathrm{B}$ are presented in Table 3 . The relative positions of 2M J044144 A and B from WFPC2 are also included (Todorov et al. 2010). Any errors that may be present in the distortion correction for the $\mathrm{AO}$ images are not accounted for in the uncertainties in Table 3, but they are only relevant to the measurement of relative astrometry across large distances on the detector array, such as between 2M J044144 A and 2M J044145 A. In addition, because each of the four objects was observed near the same location on the array between the two epochs, a comparison of the relative astrometry between epochs should not be affected by errors in the distortion correction.

In Todorov et al. (2010), we demonstrated that 2M J044144 A/B maintained similar relative positions between the WFPC2 observation and the first epoch of $\mathrm{AO}$ data, indicating that the two objects share similar motions, and that the candidate secondary is not a field star. Our second AO epoch further supports this result, as shown in Table 3. Meanwhile, the relative positions of $2 \mathrm{M} \mathrm{J} 044145 \mathrm{~A} / \mathrm{B}$ and the relative positions of $2 \mathrm{M}$ J044144 A/2M J044145 A also were unchanged within the uncertainties between the two AO epochs. For the first of these two pairs, if one component was motionless while the other had the same proper motion as the nearest group of Taurus members (group V; Luhman et al. 2009a), then their separation and position angle would change by 0.006 and 6.4, respectively, which is inconsistent with our measurements. Thus, 2M J044145 B shares the same motion as the primary, and hence is a Taurus member. Our astrometry is not sufficiently accurate to distinguish between a binary system and a pair of unrelated
Taurus members that have a small projected separation, but the latter is very unlikely given the low stellar density in Taurus. For 2M J044144 A and 2M J044145 A, the separation and position angle would change by 0.005 and 0.12 , respectively, if one was motionless while the other exhibited the proper motion of the nearest Taurus group. This relative motion is not detectable in our data because the position angle error is dominated by the uncertainty in the position angle of the camera. However, both objects are already known to be members of Taurus based on spectroscopy (Luhman 2006; Luhman et al. 2009a; Kraus \& Hillenbrand 2009).

\section{CANDIDATE COMPANIONS}

\subsection{Color-Magnitude Diagrams}

Our WFPC2 images have detected point sources within a few arcseconds of several of the primaries in Taurus and Chamaeleon I. Images of the candidate companions with separations less than $2^{\prime \prime}$ are shown in Figure 2. To assess the companionship of these objects, we can check whether they have the colors and magnitudes expected for members of these starforming regions. To do this, in Figure 3 we have constructed color-magnitude diagrams for all unsaturated point sources in the WFPC2 images of Taurus and Chamaeleon I. Some of the Taurus primaries were saturated in one of the bands and thus are absent from Figure 3. As noted in Section 2.1.1, unsaturated photometry is available for three additional members of Chamaeleon I beyond the 15 low-mass primaries that were targeted by WFPC2. The known members of these regions form sequences that are well-separated from most field stars. One exception is OTS 32, which appears below the sequence for Chamaeleon I. The subluminous appearance of this star has been observed previously and probably indicates that it is seen in scattered light (Luhman \& Muench 2008).

In Figure 3, we have circled the candidate companions that are within $2^{\prime \prime}$ of the primaries. One of these candidate companions, 2M J044144 B, appears within the sequence of Taurus members and is a likely companion (Todorov et al. 2010). The remaining five candidates fall below the cluster sequences and thus are probably field stars. Among objects beyond $2^{\prime \prime}$ from the primaries (uncircled points), one appears within the Chamaeleon I sequence, but its other optical and IR colors are inconsistent with a cool object and suggest that it is a field star. We have also marked in the color-magnitude diagrams the components of the two partially resolved binaries from Figure 1. Both components of 2MASS J04221332+1934392 appear within the Taurus sequence but the fainter component of ISO 217 is below the Chamaeleon I sequence. The anomalously blue color of ISO $217 \mathrm{~B}$ could indicate that it is background field star or may result from the large uncertainties in its photometry. We note that all of the candidate companions from Kraus \& Hillenbrand (2012) that are in our WFPC2 images appear below the member sequence in Figure 3, and hence are probably field stars. The primaries for these candidates consist of 2MASS J04152409+2910434, 2MASS J04221644+2549118 (CFHT-14), 2MASS J04302365+2359129 (CFHT-16), 2MASS $\mathrm{J} 04311907+2335047$, and 2MASS J04334291+2526470.

\subsection{Probability of Companionship}

We now examine the probability of companionship for the three candidates that have photometry consistent with membership in Taurus and Chamaeleon I (marginally consistent in the case of ISO $217 \mathrm{~B}$ ). Todorov et al. (2010) considered 


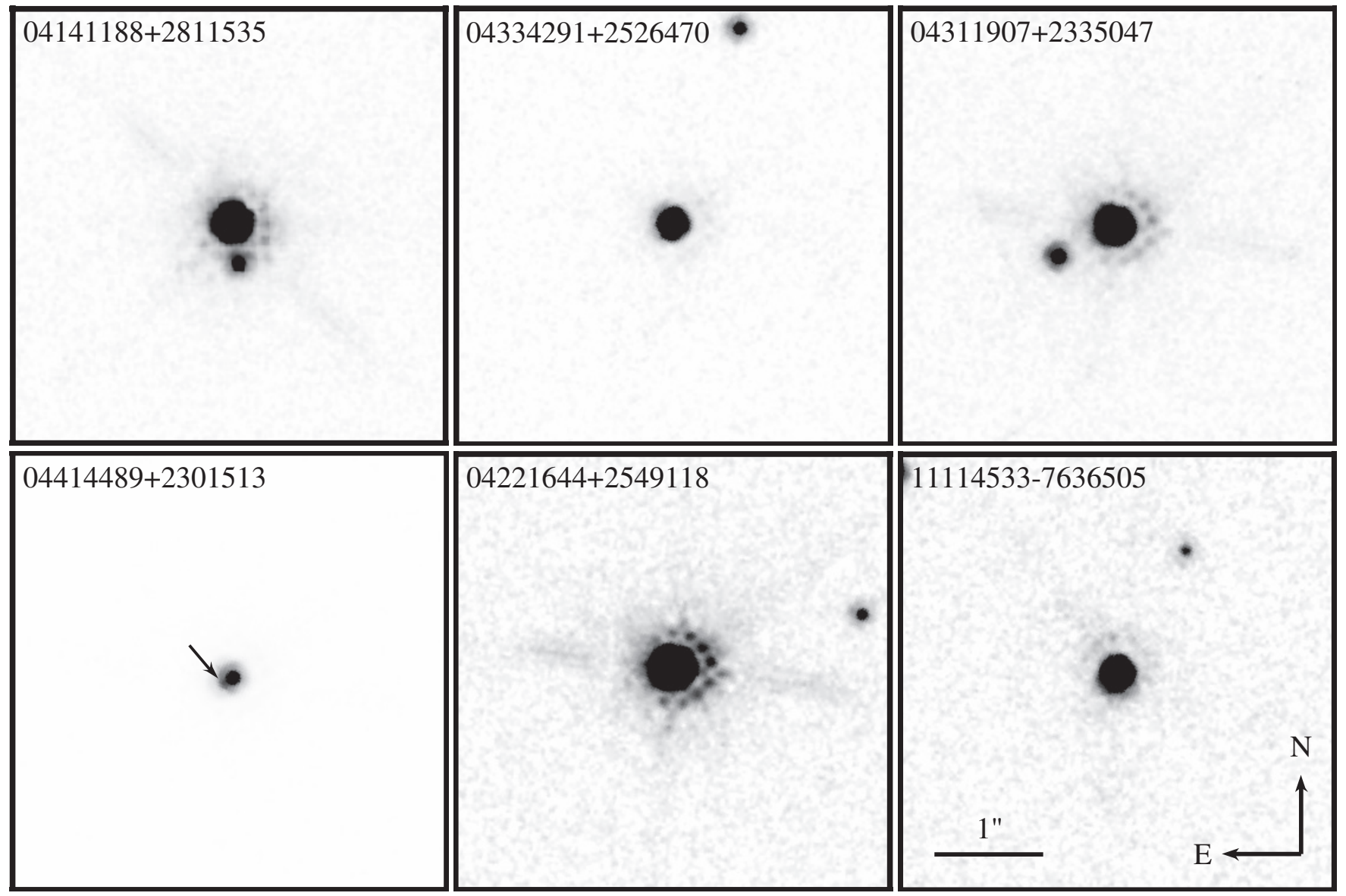

Figure 2. WFPC2 F791W images of young late-type members of Taurus and Chamaeleon I that have resolved candidate companions within $2^{\prime \prime}$. The photometry of the object near 2M J044144 is consistent with that expected for a member of Taurus while the remaining candidates are probably field stars, as shown in Figure 3. PSF-subtracted images of the companion to 2M J044144 were presented by Todorov et al. (2010).

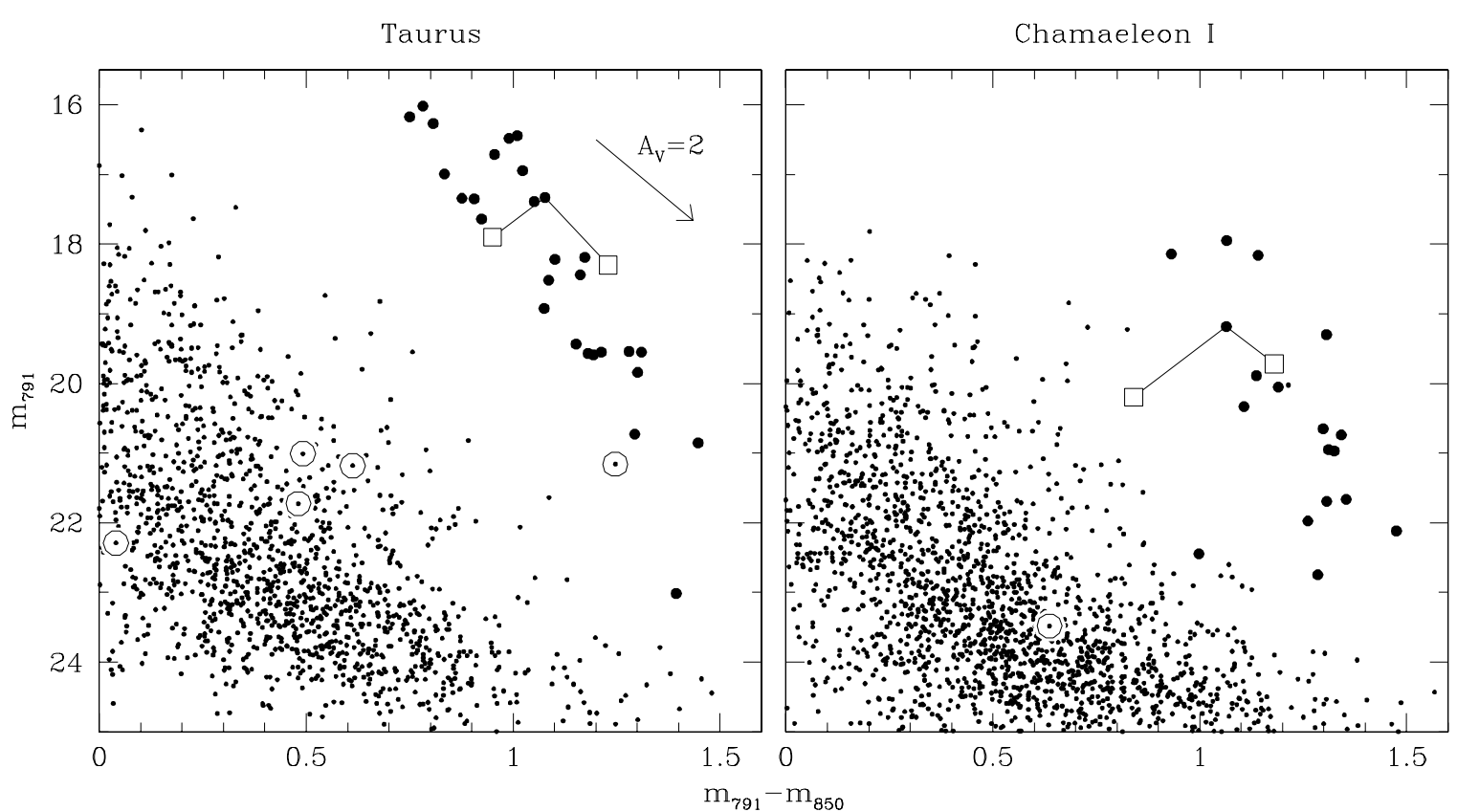

Figure 3. Color-magnitude diagrams constructed from WFPC2 images of late-type members of the Taurus and Chamaeleon I star-forming regions. We show the known members of these regions that are within the images and are not saturated (large filled circles). Among the remaining point sources (points), we indicate the ones that are within $2^{\prime \prime}$ of a known member (circles). One of these candidate companions appears within the Taurus sequence and was confirmed as a member based on its proper motion (2M J044144; Todorov et al. 2010) while the other candidates are probably field stars based on their locations below the sequences of known members. We also plot the positions of the components of the partially resolved binaries from Table 2 and Figure 1 (squares). The secondary for the pair in Chamaeleon I is bluer than expected for a cluster member, but its photometry is uncertain. 
Table 4

Late-type Targets of Multiplicity Surveys in Taurus, Cha I, and Upper Sco

\begin{tabular}{lcccc}
\hline \hline 2MASS $^{\mathrm{a}}$ & Other Name & Spectral Type & $\begin{array}{c}\rho^{\mathrm{b}} \\
(\operatorname{arcsec})\end{array}$ & Reference \\
\hline J04141188+2811535 & $\ldots$ & M6.25 & $<0.03,<0.053$ & 1,2 \\
J04151471+2800096 & KPNO 1 & M8.5 & $<0.03$ & 3 \\
J04152409+2910434 & $\ldots$ & M7 & $<0.03,<0.051$ & 1,2 \\
J04161210+2756385 & $\ldots$ & M4.75 & $<0.03$ & 4 \\
J04161885+2752155 & $\ldots$ & M6.25 & $<0.03,<0.078$ & 1,2 \\
\hline
\end{tabular}

Notes.

a 2MASS Point Source Catalog (Skrutskie et al. 2006).

b Separations measured for resolved binaries and the detection limits for unresolved sources. The adopted limits are described in Section 4.4.

References. (1) This work; (2) Kraus \& Hillenbrand 2012; (3) Kraus et al. 2006; (4) Konopacky et al. 2007; (5) Luhman et al. 2009b; (6) Todorov et al. 2010; (7) Luhman 2004a; (8) Lafrenière et al. 2008; (9) Ahmic et al. 2007; (10) Luhman 2007; (11) Neuhäuser et al. 2002; (12) Biller et al. 2011; (13) Kraus et al. 2005.

(This table is available in its entirety in a machine-readable form in the online journal. A portion is shown here for guidance regarding its form and content.)

the companionship of $2 \mathrm{M} \mathrm{J044144} \mathrm{B,} \mathrm{which} \mathrm{has} \mathrm{a} \mathrm{separation}$ of 0 '. 105 from its primary. As explained in that study, the probability that a field star with the color and magnitude of a Taurus member would appear within $0^{\prime \prime} 1$ of any of the 32 Taurus primaries in our survey is $\sim 10^{-5}$ (the astrometry from Section 3.4 also indicates that 2M J044144 B is a Taurus member rather than a field star). Given that 2MASS J04221332+1934392 $\mathrm{B}$ has a separation of 0 '.05, the probability that it is a field star is even lower. Although ISO $217 \mathrm{~B}$ is below the cluster sequence for Chamaeleon I, the probability that it is a field star is not significantly higher than these values in Taurus because the surface density of field stars is low in its vicinity of the color-magnitude diagram in Figure 3. The unresolved spectroscopy of ISO 217 A+B from Luhman (2004b) provides additional constraints on the nature of the candidate secondary. If ISO $217 \mathrm{~B}$ is a field star, it likely would be a reddened early-type star or K giant. Since ISO 217 B contributes roughly one-third of the optical flux of the pair, a field star of this kind probably would have been noticeable in the unresolved spectrum. For instance, the molecular bands of the primary would appear heavily diluted and veiled by the relatively featureless continuum of a warmer field star. Finally, because of the low stellar densities in Taurus and Chamaeleon I, the components of these three pairs are unlikely to be unrelated clusters members that have small projected separations (Luhman 2004a; Luhman et al. 2009b). Therefore, we conclude that 2M J044144 and 2MASS J04221332+1934392 likely comprise binary systems. For ISO 217 , we probably have detected either a binary companion or a jet (see the next section).

\subsection{Properties of Candidate Companions}

In Todorov et al. (2010), we estimated the physical properties for one of the three companions that we have uncovered with WFPC2, 2M J044144 B. Its projected separation of 0 .' 105 corresponds to $15 \mathrm{AU}$ at the distance of Taurus and its WFPC2 fluxes imply a mass of 5-10 $M_{\text {Jup }}$ based on theoretical evolutionary models. We now examine the properties of the other two candidates, 2MASS J04221332+1934392 B and ISO $217 \mathrm{~B}$. Their projected separations of $0{ }^{\prime} .051$ and $0^{\prime \prime} 031$ correspond to 7 and $5 \mathrm{AU}$, respectively, at the distances of Taurus and Chamaeleon I. As an unresolved pair, ISO $217 \mathrm{~A}+\mathrm{B}$ exhibits strong $\mathrm{H} \alpha$ emission (Luhman 2004b; Muzerolle et al. 2005), forbidden emission lines (Scholz \& Jayawardhana 2006), and mid-IR excess emission (Apai et al. 2005; Luhman et al. 2005a, 2008), indicating the presence of active accretion and a circumstellar disk around at least one component. In fact, a jet has been detected from ISO 217 (Whelan et al. 2009), which has a similar position angle as our candidate companion. Thus, it is possible that we have detected emission from this jet rather than a companion, although our filters should encompass little line emission from a jet. We treat ISO 217 as a binary for the purposes of this study. Meanwhile, the components of 2MASS J04221332+1934392 probably have roughly similar masses given that the flux ratio at F850LP is near unity. Neither component appears to have a close-in circumstellar disk based on the absence of mid-IR excess emission (Luhman et al. 2010).

\subsection{Binary Statistics}

To characterize the multiplicity of low-mass stars and brown dwarfs in Taurus and Chamaeleon I, we combine the results from our survey with those from previous high-resolution images in these regions. The latter were collected with WFPC2 (Kraus et al. 2006), Keck speckle imaging (Konopacky et al. 2007), and Keck AO imaging (Kraus \& Hillenbrand 2012) in Taurus and with WFPC2 (Neuhäuser et al. 2002), the Advanced Camera for Surveys on Hubble (Luhman 2007), and AO at the Very Large Telescope (Ahmic et al. 2007; Lafrenière et al. 2008) in Chamaeleon I. For comparison to these two regions, we also have compiled binary data measured for late-type members of the Upper Sco association ( $\tau \sim 11$ Myr; Pecaut et al. 2012) with WFPC2 and Keck AO (Kraus et al. 2005; Biller et al. 2011; Kraus \& Hillenbrand 2012). ${ }^{18}$ We consider data for primaries with spectral types of $\geqslant \mathrm{M} 4\left(\lesssim 0.3 M_{\odot}\right)$ using the classifications adopted by Luhman (2008) and Luhman et al. (2010, 2012). For LH 0419+15, which was observed by Kraus et al. (2006), we adopt a type of M6 (K. Luhman, in preparation). The resulting samples contain 85,66 , and 50 primaries in Taurus, Chamaeleon I, and Upper Sco, respectively, and are compiled in Table 4.

18 We have omitted USco CTIO 132 and USco CTIO 137 since they appear to be field dwarfs (Muzerolle et al. 2003; K. Luhman, in preparation).

Table 5

Low-mass Binary Fractions ( $>10 \mathrm{AU})$ in Young Regions

\begin{tabular}{lcccc}
\hline \hline Spectral Type & Taurus $^{\mathrm{a}}$ & Cha I $^{\mathrm{b}}$ & U Sco $^{\mathrm{c}}$ & Total \\
\hline M4-M6 & $7 / 39=0.18_{-0.04}^{+0.08}$ & $4 / 39=0.1_{-0.03}^{+0.07}$ & $3 / 15=0.2_{-0.06}^{+0.14}$ & $14 / 93=0.15_{-0.03}^{+0.05}$ \\
$>$ M6 & $2 / 46=0.04_{-0.01}^{+0.05}$ & $1 / 27=0.04_{-0.01}^{+0.07}$ & $1 / 35=0.03_{-0.01}^{+0.06}$ & $4 / 108=0.04_{-0.01}^{+0.03}$ \\
\hline
\end{tabular}

Notes.

a Kraus et al. (2006), Konopacky et al. (2007), Kraus \& Hillenbrand (2012), and This work.

b Neuhäuser et al. (2002), Ahmic et al. (2007), Luhman (2007), Lafrenière et al. (2008), and This work.

${ }^{c}$ Kraus et al. (2005), Biller et al. (2011), and Kraus \& Hillenbrand (2012). 
These regions have similar distances $(d \sim 150 \mathrm{pc})$ and have been observed with similar angular and mass detection limits, which allows a direct comparison of their data.

In Table 5, we present the fractions of primaries in the high-resolution imaging surveys of Taurus, Chamaeleon I, and Upper Sco for which probable companions have been detected at $>10 \mathrm{AU}$, which is the smallest separation that all of these surveys reached. Separate fractions are shown for M4-M6 $\left(0.1-0.3 M_{\odot}\right)$ and $>$ M6 $\left(\lesssim 0.1 M_{\odot}\right)$ so that we can examine the dependence of the binary fractions on spectral type, and hence stellar mass. To illustrate the distribution of separations and how it varies with the spectral type of the primary, we plot the separations of resolved binaries and the separation limits for equal-magnitude pairs among the unresolved primaries versus spectral type in Figure 4. For this diagram, we have adopted separation limits of 0 '.03 for the PC, Advanced Camera, and speckle data, 0'.06 for the WFC and NICMOS data, 0'.08 for the AO data from the Very Large Telescope, and the FWHM of the Keck AO data. We note that some AO data are capable of detecting binaries with separations that are smaller than the FWHM (Kraus \& Hillenbrand 2012).

\section{DISCUSSION}

Taurus, Chamaeleon I, and Upper Sco contain the largest samples of young low-mass stars and brown dwarfs that have been imaged at high resolution, providing the best available statistical constraints on low-mass multiplicity at ages of $\lesssim 10$ Myr. We first examine the dependence of the binary fractions in Table 5 on spectral type of the primary. For each of the three regions, the wide binary fraction $(>10 \mathrm{AU})$ is significantly lower at $>\mathrm{M} 6$ than at M4-M6. Trends of this kind have been detected previously in subsets of the data we have compiled (Kraus et al. 2005, 2006; Biller et al. 2011; Kraus \& Hillenbrand 2012), in samples of members of Taurus, Chamaeleon I, and Upper Sco at higher masses (Lafrenière et al. 2008; Kraus \& Hillenbrand 2009), and in the solar neighborhood (Burgasser et al. 2007, references therein). Given the youth and low density of the regions in question, particularly Taurus, this dependence on spectral type is very likely primordial rather than due to dynamical interactions among members of each region.

We also can examine the data in Table 5 for differences among Taurus, Chamaeleon I, and Upper Sco. For each range of spectral types, the binary fractions do not show any statistically significant differences between the regions. Comparing these binary fractions to data for field stars and brown dwarfs is more problematic because it is difficult to ensure that young and old samples encompass the same ranges of primary masses, and because a given young cluster may not represent the predominant star-forming environment for the field. Nevertheless, it is useful to compare the frequency of the widest brown dwarf binaries. Taurus and Chamaeleon I each contain one known binary brown dwarf with a separation greater than $100 \mathrm{AU}$ (Luhman 2004a; Luhman et al. 2009b). Although the Upper Sco sample that we have defined for Table 5 does not have any pairs wider than $100 \mathrm{AU}$, a few examples have been found among other brown dwarfs in the association (Allers 2006; Jayawardhana \& Ivanov 2006; Close et al. 2007; Luhman et al. 2007; Béjar et al. 2008). Thus, the data for Taurus, Chamaeleon, and Upper Sco indicate a binary fraction of a few percent for these wide binary brown dwarfs. In comparison, only one pair of this kind has been found among the several hundred known late-L and T dwarfs in the field (Burningham et al. 2010; Scholz 2010). This implies that dynamical interactions with cluster members or field

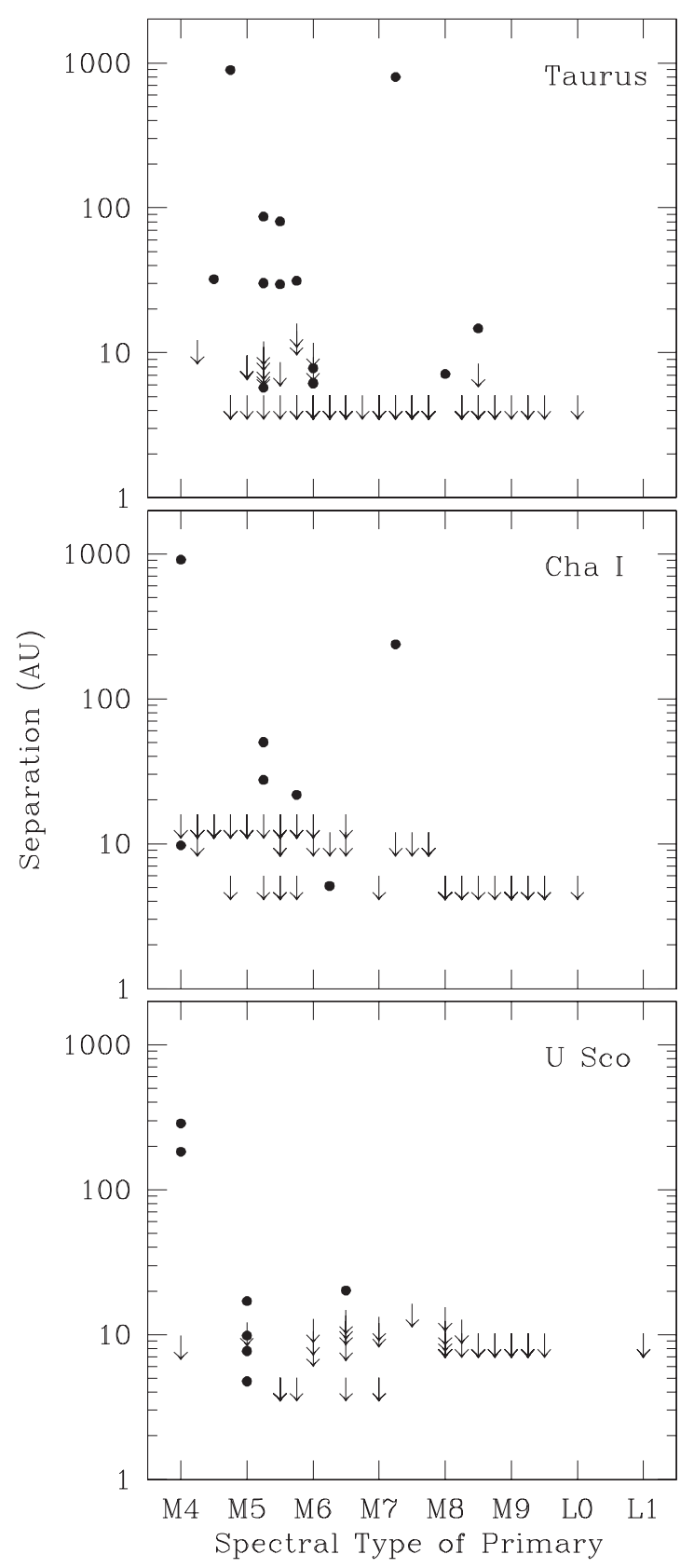

Figure 4. Binary measurements for late-type members of Taurus, Chamaeleon I, and Upper Sco (Neuhäuser et al. 2002; Kraus et al. 2005, 2006; Konopacky et al. 2007; Lafrenière et al. 2008; Biller et al. 2011; Kraus \& Hillenbrand 2012; This work). We show the projected separations of resolved binaries (points) and the detection limits for unresolved sources (arrows) as a function of the spectral type of the primary.

stars at $>10$ Myr disrupt the widest binary brown dwarfs, or that most field brown dwarfs are born under conditions different from those in Taurus, Chamaeleon I, and Upper Sco, perhaps in denser clusters where very wide binaries are disrupted or prevented from forming.

We thank Allison Youngblood, Steven Mohammed, Ijeoma Ekeh, and Jaclyn Payne for assistance with the data analysis. We acknowledge support from grant AST-0544588 from the National Science Foundation and grants GO-11203, GO-11204, and GO-11983 from the Space Telescope Science Institute. D.A. also acknowledges support from grant NNX11AG57G from the NASA Origins of Solar Systems program. The Gemini 
data were obtained through program GN-2011A-Q-10. Gemini Observatory is operated by AURA under a cooperative agreement with the NSF on behalf of the Gemini partnership: the NSF (United States), the Particle Physics and Astronomy Research Council (United Kingdom), the National Research Council (Canada), CONICYT (Chile), the Australian Research Council (Australia), CNPq (Brazil) and CONICET (Argentina). The Center for Exoplanets and Habitable Worlds is supported by the Pennsylvania State University, the Eberly College of Science, and the Pennsylvania Space Grant Consortium. MultiDrizzle is a product of the Space Telescope Science Institute, which is operated by AURA for NASA.

\section{REFERENCES}

Ahmic, M., Jayawardhana, R., Brandeker, A., et al. 2007, ApJ, 671, 2074 Allers, K. N. 2006, PhD thesis, Univ. of Texas, Austin

Apai, D., Pascucci, I., Bouwman, J., et al. 2005, Sci, 310, 834

Bate, M. R., \& Bonnell, I. A. 2005, MNRAS, 356, 1201

Béjar, V. J. S., Zapatero Osorio, M. R., Pérez-Garrido, A., et al. 2008, ApJL, 673 , L185

Biller, B., Allers, K., Liu, M., Close, L. M., \& Dupey, T. 2011, AJ, 730, 39

Bouy, H., Brandner, W., Martín, E. L., et al. 2003, AJ, 126, 1526

Burgasser, A. J., Kirkpatrick, J. D., Reid, I. N., et al. 2003, ApJ, 586, 512

Burgasser, A. J., Reid, I. N., Siegler, N., et al. 2007, in Protostars and Planets V, ed. V. B. Reipurth, D. Jewitt, \& K. Keil (Tucson, AZ: Univ. Arizona Press), 427

Burningham, B., Leggett, S. K., Lucas, P. W., et al. 2010, MNRAS, 404, 1952

Chauvin, G., Lagrange, A.-M., Dumas, C., et al. 2004, A\&A, 425, L29

Close, L. M., Siegler, N., Freed, M., \& Biller, B. 2003, ApJ, 587, 407

Close, L. M., Zuckerman, B., Song, I., et al. 2007, ApJ, 660, 1492

Diolaiti, E., Bendinelli, O., Bonaccini, D., et al. 2000, Proc. SPIE, 4007, 879

Duchêne, G., \& Kraus, A. 2013, ARA\&A, 51, 269

Hodapp, K. W., Jensen, J. B., Irwin, E. M., et al. 2003, PASP, 115, 1388

Jayawardhana, R., \& Ivanov, V. D. 2006, Sci, 313, 1279

Kenyon, S. J., Gómez, M., \& Whitney, B. A. 2008, in ASP Monograph Ser. 4, Handbook of Star Forming Regions, Vol. 1, The Northern Sky, ed. B. Reipurth (San Francisco, CA: ASP), 405

Koekemoer, A. M., Fruchter, A. S., Hook, R. N., \& Hack, W. 2002, in The 2002 HST Calibration Workshop: Hubble after the Installation of the ACS and the NICMOS Cooling System, ed. S. Arribas, A. Koekemoer, \& B. Whitmore (Baltimore, MD: STScI), 337

Koerner, D. W., Kirkpatrick, J. D., McElwain, M. W., \& Bonaventura, N. R. 1999, ApJL, 526, L25

Konopacky, Q. M., Ghez, A. M., Rice, E. L., \& Duchêne, G. 2007, ApJ, 663,394
Kraus, A. L., \& Hillenbrand, L. A. 2009, ApJ, 703, 1511

Kraus, A. L., \& Hillenbrand, L. A. 2012, ApJ, 757, 141

Kraus, A. L., White, R. J., \& Hillenbrand, L. A. 2005, ApJ, 633, 452

Kraus, A. L., White, R. J., \& Hillenbrand, L. A. 2006, ApJ, 649, 306

Lafrenière, D., Jayawardhana, R., Brandeker, A., Ahmic, M., \& van Kerkwijk, M. H. 2008, ApJ, 683, 844

Lehár, J., Falco, E. E., Kochanek, C. S., et al. 2000, ApJ, 536, 584

Luhman, K. L. 2004a, ApJ, 614, 398

Luhman, K. L. 2004b, ApJ, 602, 816

Luhman, K. L. 2006, ApJ, 645, 676

Luhman, K. L. 2007, ApJS, 173, 104

Luhman, K. L. 2008, in ASP Monograph Ser. 5, Handbook of Star Forming Regions, Vol. 1, The Southern Sky, ed. B. Reipurth (San Francisco, CA: ASP), 169

Luhman, K. L., Allen, L. E., Allen, P. R., et al. 2008, ApJ, 675, 1375

Luhman, K. L., Allen, P. R., Espaillat, C., Hartmann, L., \& Calvet, N. 2010, ApJS, 186, 111

Luhman, K. L., Allers, K. N., Jaffe, D. T., et al. 2007, ApJ, 659, 1629

Luhman, K. L., Lada, C. J., Hartmann, L., et al. 2005a, ApJL, 631, L69

Luhman, K. L., \& Mamajek, E. E. 2012, ApJ, 758, 31

Luhman, K. L., Mamajek, E. E., Allen, P. R., \& Cruz, K. L. 2009a, ApJ, 703,399

Luhman, K. L., Mamajek, E. E., Allen, P. R., Muench, A. A., \& Finkbeiner, D. P. 2009b, ApJ, 691, 1265

Luhman, K. L., McLeod, K. K., \& Goldenson, N. 2005b, ApJ, 623, 1141

Luhman, K. L., \& Muench, A. A. 2008, ApJ, 684, 654

Martín, E. L., Barrado y Navascués, D., Baraffe, I., Bouy, H., \& Dahm, S. 2003, ApJ, 594, 525

Martín, E. L., Brandner, W., \& Basri, G. 1999, Sci, 283, 1718

Muzerolle, J., Hillenbrand, L., Calvet, L., Briceño, C., \& Hartmann, L. 2003, ApJ, 592, 266

Muzerolle, J., Luhman, K. L., Briceño, C., Hartmann, L., \& Calvet, N. 2005, ApJ, 625, 906

Neuhäuser, R., Brandner, W., Alves, J., Joergens, V., \& Comerón, F. 2002, A\&A, 384, 999

Pecaut, M. J., Mamajek, E. E., \& Bubar, E. J. 2012, ApJ, 746, 154

Reid, I. N., Gizis, J. E., Kirkpatrick, J. D., \& Koerner, D. W. 2001, AJ, 121, 489

Reipurth, B., \& Clarke, C. 2001, AJ, 122, 432

Scholz, A., \& Jayawardhana, R. 2006, ApJ, 638, 1056

Scholz, R.-D. 2010, A\&A, 510, L8

Skrutskie, M. F., Cutri, R. M., Stiening, R., et al. 2006, AJ, 131, 1163

Todorov, K., Luhman, K. L., \& McLeod, K. K. 2010, ApJL, 714, L84

van Dam, M. A., Bouchez, A. H., Le Mignant, D., et al. 2006, PASP, 118,310

Whelan, E. T., Ray, T. P., Podio, L., Bacciotti, F., \& Randich, S. 2009, ApJ, 706, 1054

Wizinowich, P. L., Le Mignant, D., Bouchez, A. H., et al. 2006, PASP, 118, 297

Yelda, S., Lu, J. R., Ghez, A. M., et al. 2010, ApJ, 725, 331 